\title{
Double-tilt in situ TEM Holder with Ultra-high Stability
}

\author{
Mingjie $\mathrm{Xu}^{1}$, Sheng Dai ${ }^{1}$, Thomas Blum ${ }^{1}$, Linze $\mathrm{Li}^{1}$, Xiaoqing Pan ${ }^{1,2}$
}

1. Department of Chemical Engineering and Materials Science, University of California Irvine, Irvine, CA, 92697, United States

2. Department of Physics and Astronomy, University of California Irvine, Irvine, CA, 92697, United States

Over the past several decades, the application of transmission electron microscopy (TEM) has expanded from static characterization to in situ observation and dynamic measurements, providing new opportunities for understanding structure-property relationships of materials. However, until recently, most of the in situ TEM probing holders were only endowed with a single-tilt function [1], creating difficulties in achieving two-dimensional lattice images at atomic resolution during in situ manipulation. This is because commercial probing components, including actuator, piezo tube scanner, and electrical feedthroughs, take too much space in a TEM holder with double tilting function. In addition, the mechanical stability of the manipulation subsystem also plays an important role in atomic scale TEM imaging. According to our previous experience based on commercial in situ probing holders, continuous vibration always takes place on the sample after the tip is landed, resulting in blurred TEM images. Further, the precision of collected signals (e.g., stress, current) during in situ property measurements is dominated by the mechanical instability since it is the major source of the noise [2]. Therefore, it was necessary and imperative to develop a double-tilt in situ TEM holder with ultrahigh stability for acquiring atomic scale structure information and precision property measurements simultaneously.

Here, we report a unique design of a compact probing subsystem, showing an ultra-high stability during in situ probing, which also enables the double tilt function in a TEM holder. The ultra-high stability and multifunctionality are realized by a unique design of seal-bearing components in an in situ TEM holder. This unique seal-bearing subsystem provides superior vibration damping and electrical insulation while still maintaining excellent vacuum sealing and small form factor. We believe this design is helpful to the future development of in situ TEM setups and may broaden the application of in situ electron microscopy.

One of the main advantages of this in situ TEM holder is the double tilting function, which is essential for obtaining atomic-scale information along specific zone axes of materials. Meanwhile, our special design of vibration damping, further guarantees the collection of high quality TEM images during dynamic stimuli. The ability to perform scanning tunneling microscopy (STM) is another critical criterion to evaluate the stability and the electrical sensitivity of a scanning probe system. By using our holder, the STM topographical information can be obtained by recording the voltage applied to the piezo tube during the scanning process as the tip maintains a constant tunneling current. In addition, up to five additional components with various functionalities, besides the tilting capability, can be further incorporated through the seal-bearing of the in-situ holder, thus broadening the range of in situ TEM investigations. For example, with the addition of a fiber optic component, the cathodoluminescence (CL) spectrum of the sample can be collected by a mounted spectrometer; reversely, optical illumination can also be applied to the sample by an external light source. 
References:

[1] Q. Zhang et al, Nature Communications, 8 (2017).

[2] D.H. Alsem et al, European Microscopy Congress 2016: Proceedings (2016).

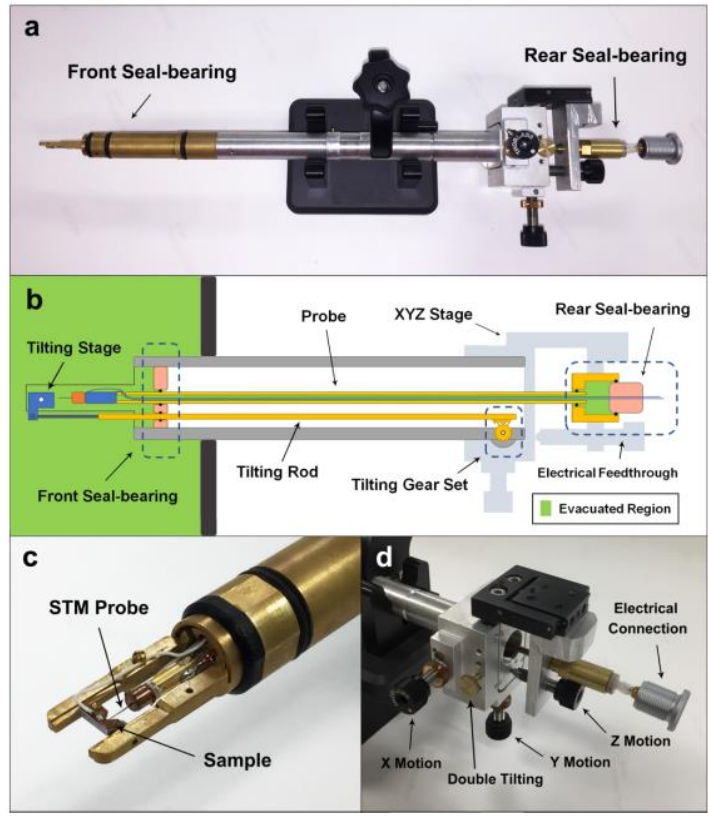

Figure 1. Photographs and schematics showing the design of double-tilt in situ TEM holder. (a) Side view photograph of the in-situ TEM holder. (b) Side-view schematic of detailed holder design. The evacuated area is marked by green color. The PEEK components are marked by pink color. (c, d) Zoomed in photographs of the front and the back end of the holder.

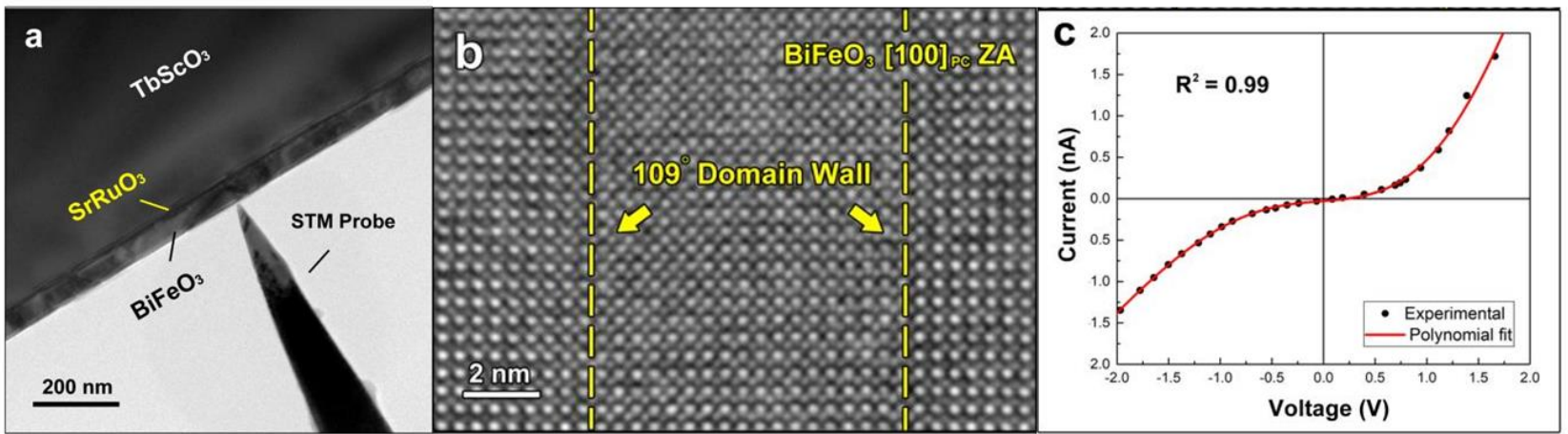

Figure 2. In situ electrical measurement of BFO/SRO/TSO cross-section sample. (a) Low magnification TEM image showing an overview of the in situ set up. (b) High quality HRTEM image taken from the BFO region showing the clear domain structure. (c) Experimental I-V sweep result of the $\mathrm{BFO} / \mathrm{SRO} / \mathrm{TSO}$ sample and polynomial fit. 